Boletim I'écnico da Divisão de Experimentação e Pesquisas I N S T I T U TO A G RONÔM I CO

Vol. 2 Campinas, Setembro de $1942 \quad$ N. 9

\title{
COEFICIENTE DE VARIAÇÃO E ÍNDICE DE VARIANÇA
}

F. G. Brieger $\left({ }^{*}\right)$

\section{INTRODUÇÃO}

$\mathrm{Na}$ experimentação em geral $e$, especialmente, na experimentação agrícola, surge a necessidade de comparar a variação de valores da mesma natureza, mas de dimensões bem diferentes. Conhecemos, por exemplo, a produção média de uma espécie por parcela de poucos metros quadrados e o seu erro "standard". Que produção esperaríamos por hectare e, qual a exatidão para tal estimativa? Ou, como poderemos, em ensaios de seleção, comparar a variação de um milho de porte baixo com a de um de porte alto?

Os dois problemas podem, mais correta e matematicamente, ser formulados da seguinte maneira:

Foi determinado, num ensaio, que uma área de a $\mathrm{m} 2$ produzia na média $\bar{a} \mathrm{~kg}$ de um produto qualquer, com um erro "standard" de $\pm \sigma_{\mathrm{a}}$. Qual a produção média de uma área $\mathbf{b}$, maior, ou tambem menor, e qual o seu erro "standard"? Se a área $\mathbf{b}$ fosse $\mathbf{m}$ vezes maior ou menor que a área a seria a sua produção e o seu erro "standard" m vezes tambem maior ou menor?

A resposta à primeira parte da pergunta é, sem dúvida, positiva. Quando dizemos que a área $\mathbf{b}$ é $\mathbf{m}$ vezes a área $\mathbf{a}$, deve-se entender que esta relação não é somente quartitativa, mas tambem qualitativa, isto é, que a área $\mathbf{b}$ se compõe de $\mathbf{m}$ parcelas a de igual qualidade e que as áreas $\mathbf{b}$ e $\mathbf{a}$ são apenas diferentes no tamanho. Na terminologia estatística dizemos que as áreas $\mathbf{a} \in \mathbf{b}$ são amostras, de tamanhos desiguais, mas pertencendo ao mesmo universo. Neste caso podemos dizer que:

$$
\begin{aligned}
& \text { se área } b=\mathrm{m} \text {. área } \underline{a} \\
& \text { produção: } \overrightarrow{\mathrm{b}}=\mathrm{m} \text {. } \mathrm{a}
\end{aligned}
$$

(*) Da Escola Superior de Agricultura "Luiz de Queiroz". 
Mas seria necessário provar que a mesma relação existe tambem para o erro "standard", isto é :

$\sigma_{\mathbf{b}}=\mathrm{m} . \sigma_{\mathrm{a}}$ ?

outro problema é o seguinte: se tivermos obtido num experimento uma média qualquer $\bar{v}$ e um erro "standard" $\pm \sigma$, poderemos reduzir este último a um valor absoluto e constante, formando, por exemplo, 0 quociente $\sigma: \bar{v} ;$ se se argumentar que o erro é $\pm \sigma$ em volta da média $\vec{v}$, o erro em volta da unidade absoluta deve ser então $\sigma: \overline{\mathbf{v}}$. trar.

Estas duas questões são intimamente ligadas, como é facil demons-

Suponhamos que temos três áreas, ou três variedades, sendo as médias :

$$
\overline{\mathrm{a}}=\frac{\overline{\mathrm{b}}}{\mathrm{m}}=\frac{\overline{\mathrm{c}}}{\mathrm{n}} .
$$

De acordo com a nossa hipótese, deveria ser :

$$
\sigma_{\mathrm{r}}=\frac{\sigma_{\mathrm{b}}}{\mathrm{m}}=\frac{\sigma_{\mathrm{c}}}{\mathrm{n}}
$$

ou então, dividindo as equações e cancelando os fatores $\mathrm{m}, \mathrm{n}$

$$
\frac{\sigma_{\mathrm{a}}}{\overline{\mathrm{a}}}=\frac{\sigma_{\mathrm{b}}}{\overline{\mathrm{b}}}=\frac{\sigma_{\mathrm{c}}}{\overline{\mathrm{c}}}=\text { constante. }
$$

Assim parece que o ponto principal do nosso argumento será a prova da equação final :

$$
\frac{\text { erra }}{\text { média }}=\frac{\sigma}{\overline{\mathrm{v}}}=\text { constante ou } \frac{\sigma}{\overline{\mathrm{v}}} \cdot 100=\sigma \%=\text { constante. }
$$

De-fato, não encontramos nenhuma prova na literatura estatística, e mostraremos adiante que se trata apenas de aproximação permitida em certos casos.

É atualmente facil mostrar que o conceito de um coeficiente de variação constante é teoricamente sem fundamento. Se temos a equação $\overrightarrow{\mathrm{b}}=\mathrm{m} \cdot \overline{\mathrm{a}}$, podemos considerar $\mathrm{b}$ como uma soma :

$$
\begin{aligned}
& \vec{b}=\bar{a}+\bar{a}+\ldots \ldots(\mathrm{m}) . \\
& O \text { erro de } \bar{b} \text { é, então, o erro de uma soma, e teremos: } \\
& \sigma_{b}= \pm \sqrt{\sigma_{a}^{2}+\sigma_{a}^{2} \ldots(m)}= \pm \sigma_{a} \sqrt{\mathrm{m}}
\end{aligned}
$$

e igualmente:

$$
\sigma_{\mathrm{c}}= \pm \sqrt{\sigma_{\mathrm{a}}^{2}+\sigma_{\mathrm{a}}^{2} \ldots(\mathrm{n})}= \pm \sigma_{\mathrm{a}} \sqrt{\mathrm{n} .}
$$

Finalmente, teremos:

$$
\sigma_{\mathrm{a}}=\frac{\sigma_{\mathrm{b}}}{\sqrt{\mathrm{m}}}=\frac{\sigma_{\mathrm{c}}}{\sqrt{\mathrm{n}}}=\text { constante. }
$$


Igualmente, poderemos exprimir, em forma de soma, a redução de uma média à unidade :

$$
\overline{\mathrm{v}}=1+1+1 \ldots(\overline{\mathrm{v}}) \text {. }
$$

Teremos assim a relação entre o erro da amostra $\sigma$ e o erro da unidade $\sigma_{1}$ :

$$
\sigma= \pm \sqrt{\sigma_{1}^{2}+\sigma_{1}^{2} \ldots(\bar{v})}= \pm \sqrt{\sigma_{2}^{2} \cdot \bar{v}}= \pm \sigma_{1} \sqrt{\bar{v}}
$$

ou : $\quad \sigma_{1}=\frac{\sigma}{\sqrt{\overrightarrow{\mathrm{v}}}}=$ constante.

Este termo $\sigma: \sqrt{\overline{\mathrm{v}}}=\sqrt{\sigma^{2}: \overline{\mathrm{v}}}$ chamaremos, em seguida, Indice da variança $\left(^{*}\right)$.

A única premissa feita na derivação das fórmulas é que a aplicação da fórmula do erro de uma soma é legítima, isto é, que podemos considerar as áreas $b$ e $c \ldots$ quantitativa e tambem qualitativamente como múltiplos de uma mesma área $a_{s}$ e a média $\overline{\mathrm{v}}$ como múltiplo da unidade.

Devemos fazer então uma distinção entre esta premissa e o teorema estatístico representado pela fórmula $\frac{\sigma}{\sqrt{\frac{\bar{v}}{\mathrm{v}}}}=$ constante. Este teorema, depois da derivação acima, não precisa mais ser provado, mas a premissa depende ainda da homogeneidade do material em cada experimento e exige uma comprovação experimental. Nos capítulos subsequentes daremos esta prova.

Um índice de variança constante indica que a premissa estava certa ; uma inconstância mostra, ao contrário, que estava errada, e que reunimos amostras heterogêneas.

Ao mesmo tempo, os resultados destes experimentos demonstrarão que o coeficiente de variação não é um valor constante, mas sim uma função matemática da média.

\section{HOMOGENEIDADE DO TERRENO}

Em todos os ensaios agrícolas encontraremos, inicialmente, sempre - mesmo requisito: a área experimental deve ser bastante homogênea. Os métodos modernos dos planos básicos, como blocos ao acasó, quadrado latino, etc., teem por fim provar se havia homogeneidade $e$, no caso contrário, permitir a eliminação estatística do efeito da heterogeneidade.

Mas, de modo geral, e mesmo em áreas bem escolhidas, quase sempre há certa heterogeneidade do terreno. A área total do experimento

(*) Na literatura inglesa é comum usar-se o quadrado do erro "standard", termo este designado como "variance", que traduzimos por varianca. 
não é uma soma de parcelas muito equivalentes. Assim podemos esperar, com menos razão ainda, que uma área maior e não selecionada seria um simples múltiplo da área básica da parcela.

Uma vez que incluimos na área maior tanto manchas melhores como piores do que as parcelas experimentais, poderemos argumentar, com certa probabilidade, que a produção média da área maior seja simplesmente um múltiplo da produção da área menor. A área maior, porem, vai sempre ser mais variavel do que a parcela, e o erro não cresce proporcionalmente. Em forma matemática podemos assim contrastar as fórmulas ideais com as empíricas :

\section{Fórmula ideal}

área $\mathrm{b}=\mathrm{m} \cdot \mathrm{a}$

produção $\bar{b}=m \cdot \bar{a}$

Erro "standard"

da distribuição $\sigma_{b}=\sigma_{\mathfrak{a}} / \bar{m}$

\section{Fórmula empírica}

$$
\begin{aligned}
& b=m \cdot a \\
& \vec{b}=m \cdot \vec{a}
\end{aligned}
$$$$
\sigma_{\mathrm{b}} \geq \sigma_{\mathrm{a}} \sqrt{\mathrm{m}}
$$

Assim, chegamos à conclusão de que a heterogeneidade das terras não permite a aplicação das fórmulas ideais. Querendo generalizar conclusões obtidas em experiências limitadas, podemos concluir que a produção média é aproximadamente proporcional às áreas usadas, mas não temos possibilidades de avaliar com segurança se essa aproximação é satisfatória ou não. Em nenhum caso, portanto, podemos tirar conclusão exata ou bastante aproximada sobre o comportamento do erro "standard" da distribuição na área maior. Diremos apenas que, pelo menos, os índices de variança das diferentes áreas podem ser iguais, $\mathrm{e}$, os das áreas maiores, geralmente maiores do que os das menores.

\section{COEFICIENTE DA VARIAÇÃO E ÍNDICE DA VARIANÇA}

Para o caso anterior, havia amplo material de conhecimento geral, representado por grande número de ensaios agrícolas com repetições, que permitem avaliação da heterogeneidade de terreno e que dispensam mais exemplos. Porem, para provar a constância do índice de variança e a inconstância do coeficiente de variação, daremos alguns exemplos com os resultados de seis séries experimentais : uma com café, duas com laranja e, finalmente, três de medidas de uma experiência de milho.

Os resultados estão reunidos nos respectivos quadros I a III e ilus. trados nos gráficos 1 a 6 .

Nos quadros encontramos sempre a mesma organização. A primeira coluna contem o número caraterístico das amostras, a segunda o número de indivíduos por amostra e, nas colunas seguintes, a média, o erro "standard" da distribuição, o coeficiente da variação e o índice da variança, sempre por amostra.

A prova da constância do quociente $\sigma: \sqrt{\overrightarrow{\mathrm{v}}}$ consiste num teste "entre-dentro". Como indicado nos quadros I a III, as médias foram 
reunidas em classes de intervalos iguais. Foram depois calculados tambem os índices da variança por classe. Assim, poderemos finalmente comparar a variação dos índices médios com a dos índices individuais, em volta do índice médio de cada classe. Se essas duas componentes forem iguais, poderemos concluir que a variação dos índices é independente da das médias por amostra, e que o índice da variança poderá ser considerado como razoavelmente constante, sujeito apenas a pequenas variações do livre jogo do acaso. Os resultados do teste figuram no quadro IV.

\section{1) Experimento de café :}

Produção em kg por planta. Quadro I e gráfico l. As denominações na primeira coluna são explicadas no trabalho de Brieger (1).

As médias variam desde $0,088 \mathrm{~kg}$ até o máximo de $12,5 \mathrm{~kg}$. Os erros "standard" crescem proporcionalmente com as médias, e a curva do coeficiente da variação não corresponde a uma linha reta e horizontal, não sendo, portanto, o coeficiente constante e independente das médias. $O$ índice da variança acompanha razoavelmente uma linha reta e horizontal e o teste "entre-dentro" dá um quociente insignificante de $\vartheta=1,47$.

$O$ índice é, evidentemente, constante e atinge o valor de 1,20.

\section{2) Experimento de laranja Baianinha :}

Número de frutas por árvore. Gráfico 5. As designações na primeira coluna do quadro são explicadas na publicação de Brieger, Moreira e Leme (2).

A situação é bem semelhante à do caso anterior. $O$ valor menor das médias alcançado é de 18 e o máximo é de 167 frutas por árvore.

O erro "standard" parece crescer e o coeficiente da variação decrescer com o aumento do valor das médias. $\bigcirc$ polígono do índice da variança acompanha razoavelmente uma linha reta e o teste "entredentro" dá um valor insignificante de $\vartheta=1,64$.

Evidentemente, o índice da variança é constante e tem o valor médio de 5,97.

\section{3) Experimento da influência do cavalo sobre o cavaleiro (la- ranja Baianinha):}

Número de frutas por árvore. Gráfico 6. Os nomes na primeira coluna indicam o cavalo usado e o ano da colheita. Os dados com pormenores serão publicados dentro em breve por Brieger e Moreira. Compare Moreira (3).

Os valores extremos das médias atingem 1,8 e 360 frutas por árvore. Como nos dois casos anteriores, os valores dos erros "standard" crescem com as médias, enquanto que os coeficientes da variação diminuem, ao mesmo tempo, de $275 \%$ até $28 \%$. 
De outro lado, o índice da variança de 5,32 é razoavelmente constante, como demonstra o teste "entre-dentro", com o valor de $\vartheta=1,33$.

\section{4) Experimento de milho :}

Noventa famílias da geração $\mathrm{F}_{3}$ de um cruzamento entre milho brasileiro e estrangeiro foram analisadas em várias direções (Brieger, não publicado). Referimo-nos aquí apenas a três caracteres : altura da espiga, do chão até a sua base : altura da planta, do chão até a base da flecha : florescimento, isto é, o número de dias entre semeação e aparecimento da barba.

Altura da espiga - Gráfico 2. As médias por família variam desde $5,7 \mathrm{~cm}$ até $44 \mathrm{~cm}$. Como nos casos anteriores, tanto o erro "standard" como o coeficiente da variação não são constantes. De outro lado, o índice da variança varia insignificantemente, em volta de um valor de 2,37, como demonstrado pelo teste "entre-dentro" e o gráfico.

Altura da planta - Quadro II, gráfico 3. Os valores extremos das médias parciais são $52 \mathrm{~cm}$ e $145 \mathrm{~cm}$. O erro "standard" cresce paralelamente com a média até mais ou menos o valor de $100 \mathrm{~cm}$, ficando quase constante em famílias de maior altura. O coeficiente da variação, por sua vez, decresce lentamente desde 22\% até 14\%. O índice da variança não é tão constante como nas outras classes, variando ligeiramente, mas de modo irregular, em volta de 1,92.

Florescimento --. Quadro III, gráfico 4. As médias variam de 47 até 72 dias. $O$ erro "standard" cresce com a média, de \pm 3 dias até cerca de \pm 6 dias. Mas desta vez, tanto o coeficiente de variação como índice da variança são praticamente constantes, variando os polígonos apenas ligeiramente em volta de uma linha horizontal.

Em resumo, podemos dizer que, nos 6 casos experimentalmente estudados, achamos uma conslância bem acentuada do índice de variança.

Apenas em um caso (Altura da planta) o teste "entre-dentro" apresentou variação excessiva "entre". Porem uma vez que esta era bem desorientada, podemos atribuíla à grande heterogeneidade genética do material, o qual consistia em famílias de uma geração $F_{3}$.

Mostraremos, pelo teste "entre-dentro" e pela comparação nos 6 exemplos acima, que:
1) $\stackrel{\sigma}{\frac{\sigma}{v}} \cdot 100 \neq$ constante
2) $\frac{\sigma}{\sqrt{\mathrm{v}}} \approx$ constante ou $\sigma:=\mathrm{k} \sqrt{\overline{\mathrm{v}} \text {. }}$

Isto não exclue, entretanto, a possibilidade de que poderemos achar ainda melhores soluções para exprimir matematicamente a relação entre o erro "standard" e a média. De-fato, no caso do café (1) concluiu-se que a fórmula:

$$
\sigma=\sqrt{\overline{\mathrm{v}}}-0,2 \sqrt[3]{\overline{\mathrm{v}}}^{\mathrm{s}}
$$

dava uma aproximação ainda melhor. 
No caso do experimento altura da planta foi encontrada, por interpolação gráfica, uma aproximação melhor dos dados observados, a qual difere da curva $\sigma=\mathrm{k} . V \overline{\bar{v}}$ para valores extremamente pequenos ou extremamente grandes de $\bar{v}$.

É bem possivel que a constância relativa do índice da variança fosse menos acentuada, se a variação das médias se estendesse sobre amplitude maior do que nos casos apresentados. É, de-fato, de se esperar que em tais casos a situação biológica fundamental mudasse, de tal modo que pudesse afetar a intensidade e a dimensão da variação.

Mas estas restrições não alteram fundamentalmente nada no fato de o indice da variança ser razoavelmente constante em cada um dos casos.

Para melhor considerar a variação do coeficiente da variação, dividimos os exemplos em três grupos:

Os primeiros quatro casos teem em comum que as médias parciais atingem praticamente o limite absoluto do valor zero. Aquí o coeficiente da variação é sempre altamente inconstante, aproximando-se assimptoticamente do valor $\infty$.

O último caso (florescimento do milho) representa um outro extremo. A variação é bastante afastada do limite zero, sendo a média menor de 46,7 dias e o erro de distribuição de $\pm 2,3$, de modo que não encontraremos valores individuais menores do que cerca de 40 dias. Aquí o coeficiente da variação se mostrou aproximadamente constante.

$O$ caso da altura da planta em milho representa um tipo intermediário. A média mínima é de $52,1 \mathrm{~cm}$ com um erro da distribuição de $\pm 11,2 \mathrm{~cm}$. Deste modo serão esperadas talvez plantas extremas, de $20 \mathrm{~cm}$ apenas de altura. Este valor poderá ser atingido com uma frequência de cerca de 1 em 1000 ! A-pesar-de ficarmos evidentemente afastados do limite absoluto zero, não devemos esquecer que deve existir um limite fisiológico para altura da planta e o efeito deste limite se faz sentir. Asssim, o coeficiente da variação não é nem aproximadamente constante, nem tão variavel como nos primeiros quatro casos.

Se nos voltarmos mais uma vez aos primeiros quatro exemplos, notamos que em todos eles a curva do coeficiente desce inicialmente com muita rapidez, mas começa a aproximar-se assimptoticamente de uma linha horizontal desde valores do coeficiente de cerca de $25 \%$.

Em resumo, podemos concluir que apenas o índice da variança é, defato, uma constante que serve para comparar a variação de variaveis de natureza idêntica, mas de dimensão diferente. Porem, o coeficiente da variação é aproximadamente constante apenas em alguns casos, quando se trata de valores menores do que $25 \%$ e tambem quando a dimensão das médias não é excessivamente diferente.

\section{A UTILIDADE DO COEFICIENTE DA VARIAĞ̃̃O}

Chegamos à conclusão de que o uso do coeficiente de variação não se justifica do ponto de vista teórico para comparar a variação de séries 
de variaveis de dimensão diferente. Porem com este o termo não perde de todo o seu valor.

$O$ índice da variança não é um número absoluto no sentido matemático. Não dividimos dois valores da mesma dimensão, por exemplo, um erro "standard" e uma média, ambas medidas em cm, gr, etc., mas um valor pela raiz quadrada do outro. Este índice, que é apropriado para comparar estatisticamente a variação em amostras diferentes, não nos dá idéia de dimensão da variação em cada amostra. Nesta direção o coeficiente da variação mantem a sua importância e utilidade.

Exemplifiquemos : suponhamos que, num ensaio de adubação, com quatro repetições completas, achamos um coeficiente de variação de $10 \%$. Podemos então calcular o seguinte: para poder dizer se a produção de uma parcela é ou não melhor do que as demais será necessário que ela seja diferente da produção média das parcelas no mínimo em 2,58 x $10 \%=25,8 \%$. Para a produção média das quatro parcelas de uma das adubações usadas, uma diferença mínima de $2,58 \times 10 \%: \sqrt{4}=$ $=12,4 \%$ será necessária.

Sem saber, no caso do exemplo dado, os valores atuais das produções e do erro "standard" da distribuição, podemos dizer que ele permite apenas avaliar o efeito da adubação, se esta provoca pelo menos um aumento da produção de $12,4 \%$ sobre a produção média do ensaio.

Suponhamos, enfim, que foram realizadas três séries experimentais, com 4 repetições cada uma e com 8 ou mais tratamentos, e que os coeficientes da variação fossem $5 \%, 10 \%$ e $20 \%$. Teremos, então, para os limites mínimo de $1 \%$ probabilidade, as seguintes diferenças entre produção média por tratamento e média geral :

$$
\begin{aligned}
2,58 & \text { x } 5: \sqrt{4}=6,45 \% \\
2,58 & \times \quad 10: \sqrt{4}=12,90 \% \\
2,58 & \times 20: \sqrt{4}=25,80 \%
\end{aligned}
$$

É evidente que a eficiência dos ensaios é bastante diferente. No primeiro caso já um aumento da produção de $6,5 \%$ é significante, e no último apenas um aumento de mais do que $25 \%$.

$O$ coeficiente da variação se mostrou assim como uma boa medida para avaliar rapidamente a efjciência de um ensaio.

\section{CONCLUSÃO}

1. Para comparar a variação em séries de variaveis de dimensão diferente não deve ser usado o coeficiente da variação $\sigma \%=\sigma .100 \div \overrightarrow{\mathrm{v}}$, mas o índice da variança $\sigma \div V \overline{\overline{\mathrm{v}}}$.

2. Esta tese foi comprovada por conclusões de estatística teórica.

3. Em seis casos concretos, baseados em dados experimentais, foi demonstrado que, de-fato, o índice da variança é razoavelmente constante; com o coeficiente da variação não se dá o mesmo. 
4. O coeficiente da variação torna-se apenas aproximadamente constante quando as médias são bastante afastadas do limite absoluto zero ou de outros limites biológicos, e quando as médias a serem comparadas não são diferentes demais.

5. Em experimentos agrícolas e em terras razoavelmente homogêneas, podemos supor que a produção média é proporcional à área usada, de modo que se pode calcular, por simples multiplicação, a produção por hectare, alqueire, etc.

Porem, não é possivel determinar, por simples cálculo, qual seria o erro "standard" por área maior, podendo-se apenas dizer que o índice da variança seria maior em áreas maiores.

6. O coeficiente da variação mantem a sua importância para avaliar a eficiência de um ensaio.

\section{SUMMARY}

The object of the present paper is a study of the usefulness of two relative measures of variation, the well known coefficient of variation and a new term proposed in this paper and called the index of variance. These terms are defined by the equations :

$$
\begin{aligned}
& \text { coefficient of variation: } \sigma \%=\frac{\sigma}{\overline{\mathrm{v}}} \cdot 100 \\
& \text { index of variance : } \frac{\sigma}{\sqrt{\overline{\mathrm{v}}}} .
\end{aligned}
$$

1. It is shown that, for theoretical reasons, only the index of variance may be expected to be constant. Six different experimental series actually proved this constancy, showing at the same time the variability of the coefficient of variation which proved to be dependent upon the respective mean. The coefficient of variation becomes approximately constant when the respective means are sufficiently distant from the absolute limit zero or other biological limits.

2. Thus the index of variance may be used to prove the homogeneity of variation in samples with means of different dimensions.

3. Through this it is shown that the index of variance should be constant, it is explained that for biological reasons we may not always find a good fit between the observed and the expected data, calculated by means of the equation $=\mathrm{k} \overrightarrow{\mathrm{v}}$ where $\mathbf{k}$ represents a biological constant. In two cases a better fit was obtained using more complicated formulae.

4. While it seems justified in agricultural experimentation to accept proportionality between mean yield and area, no such relation exists for the standard error. We may only say that generally the index of variance for large areas is not equal, but bigger than that for smaller ones.

5. The coefficient of variation cannot be used as a general term for comparing the variation in series of different dimensions, where we must apply the index of variance. But it still retains its value as a measure of the efficiency of experiments.

\section{LITERATURA CITADA}

1. Brieger, F. G. Análise estatística da Experiência de café Bourbon e seleção de café por métodos modernos. (Capítulo II do trabalho: Melhoramento de Coffea arabica L. var. Bourbon, de J. E. Teixeira Mendes, F. G. Brieger, C. A. Krug e A. Carvalho.) Bragantia 1:26-119, gráf. 1-32, 1941.

2. Brieger, F. G., S. Moreira e Z. Lueme. Estudo sobre o melhoramento da laranja "Baía" III. Bragantia 1:567-610, fig. 1-10b, 1941.

3. Moreira, S. Experiências de cavalos para citrus I. Bragantia 1:525-565, fig. 1-20, gráf. 1-6, 1941. 


\section{QUADRO I}

CAFÉ

Produção em kg por planta

\begin{tabular}{|c|c|c|c|c|c|}
\hline$N$ & $\mathrm{n}$ & $\bar{v}$ & $\sigma$ & $\sigma \%$ & $\sigma: \sqrt{\overline{\mathrm{v}}}$ \\
\hline $\begin{array}{rl}\text { I forte } & 39 \\
\text { I fraco } & 35 \\
\text { II forte } & 36 \\
\text { III } a & 39\end{array}$ & $\begin{array}{l}68 \\
68 \\
49 \\
50\end{array}$ & $\begin{array}{l}0,088 \\
0,424 \\
0,486 \\
0,796\end{array}$ & $\begin{array}{l}0,255 \\
0,907 \\
0,624 \\
0,942\end{array}$ & $\begin{array}{l}289,8 \\
213,9 \\
128,4 \\
118,3\end{array}$ & $\begin{array}{l}1,16 \\
0,72 \\
1,02 \\
0,95\end{array}$ \\
\hline $\begin{array}{l}\text { I forte } 35 \\
\text { I forte } 32 \\
\text { II forte } 38\end{array}$ & $\begin{array}{l}68 \\
68 \\
49\end{array}$ & $\begin{array}{l}1,190 \\
1,222 \\
1,269\end{array}$ & $\begin{array}{l}0,863 \\
0,775 \\
1,366\end{array}$ & $\begin{array}{r}72,56 \\
63,42 \\
107,64\end{array}$ & $\begin{array}{l}1,26 \\
1,30 \\
0,82\end{array}$ \\
\hline $\begin{array}{r}\text { II fraco } 3 \\
\text { I forte } 33 \\
\text { III } \\
\text { II forte } 3 \\
\text { I fraco } 3 \\
\text { I fraco } 3\end{array}$ & $\begin{array}{l}29 \\
68 \\
62 \\
49 \\
68 \\
68\end{array}$ & $\begin{array}{l}2,241 \\
2,553 \\
2,665 \\
2,667 \\
2,781 \\
2,853\end{array}$ & $\begin{array}{l}1,447 \\
0,963 \\
0,917 \\
1,685 \\
1,505 \\
0,897\end{array}$ & $\begin{array}{l}64,57 \\
37,72 \\
34,41 \\
63,18 \\
54,12 \\
31,44\end{array}$ & $\begin{array}{l}1,03 \\
1,66 \\
1,78 \\
0,97 \\
1,11 \\
1,88\end{array}$ \\
\hline $\begin{array}{l}\text { I fraco } 35 \\
\text { II fraco } 3 \\
\text { III } \\
\text { II fraco } 3\end{array}$ & $\begin{array}{l}68 \\
29 \\
62 \\
29\end{array}$ & $\begin{array}{l}3,021 \\
3,272 \\
3,653 \\
3,993\end{array}$ & $\begin{array}{l}1,440 \\
1,116 \\
1,390 \\
1,112\end{array}$ & $\begin{array}{l}47,67 \\
34,11 \\
38,05 \\
27,85\end{array}$ & $\begin{array}{l}1,21 \\
1,62 \\
1,38 \\
1,80\end{array}$ \\
\hline $\begin{array}{l}\text { III } \\
\text { II forte } 33 \\
\text { III }\end{array}$ & $\begin{array}{l}62 \\
49 \\
62\end{array}$ & $\begin{array}{l}4,452 \\
4,459 \\
4,579\end{array}$ & $\begin{array}{l}1,853 \\
1,317 \\
1,830\end{array}$ & $\begin{array}{l}41,62 \\
29,54 \\
39,97\end{array}$ & $\begin{array}{l}1,14 \\
1,60 \\
1,17\end{array}$ \\
\hline $\begin{array}{c}\text { I fraco } 3 \\
\text { III } \\
\text { II fraco } \\
\text { I forte }\end{array}$ & $\begin{array}{l}68 \\
62 \\
29 \\
68\end{array}$ & $\begin{array}{l}5,029 \\
5,569 \\
5,679 \\
5,856\end{array}$ & $\begin{array}{l}1,545 \\
2,539 \\
2,878 \\
1,963\end{array}$ & $\begin{array}{l}30,72 \\
45,59 \\
50,68 \\
33,52\end{array}$ & $\begin{array}{l}1,45 \\
0,93 \\
0,83 \\
1,23\end{array}$ \\
\hline $\begin{array}{l}\text { I fraco } 3 \\
\text { II fraco } 3 \\
\text { I forte } \\
\text { II forte } 35\end{array}$ & $\begin{array}{l}68 \\
.29 \\
68 \\
49\end{array}$ & $\begin{array}{l}7,119 \\
7,403 \\
7,471 \\
7,559\end{array}$ & $\begin{array}{l}2,168 \\
2,163 \\
2,404 \\
2,186\end{array}$ & $\begin{array}{l}30,45 \\
29,22 \\
32,18 \\
28,92\end{array}$ & $\begin{array}{l}1,23 \\
1,26 \\
1,13 \\
1,26\end{array}$ \\
\hline III $\mathrm{b}$ & 12 & 9,325 & 2,720 & 29,17 & 1,12 \\
\hline $\begin{array}{l}\text { II forte } 3 \\
\text { III } \\
\text { I forte } 38\end{array}$ & $\begin{array}{l}49 \\
62 \\
68\end{array}$ & $\begin{array}{l}10,012 \\
10,427 \\
10,763\end{array}$ & $\begin{array}{l}3,418 \\
2,698 \\
2,314\end{array}$ & $\begin{array}{l}34,14 \\
25,88 \\
21,50\end{array}$ & $\begin{array}{l}0,93 \\
1,20 \\
1,42\end{array}$ \\
\hline $\begin{array}{l}\text { I fraco } 3 \\
\text { II forte } 35 \\
\text { II fraco } 3\end{array}$ & $\begin{array}{l}68 \\
49 \\
29\end{array}$ & $\begin{array}{l}11,015 \\
11,120 \\
11,476\end{array}$ & $\begin{array}{l}2,581 \\
3,730 \\
3,426\end{array}$ & $\begin{array}{l}23,43 \\
33,54 \\
29,85\end{array}$ & $\begin{array}{l}1,29 \\
0,89 \\
0,99\end{array}$ \\
\hline II fraco 39 & 29 & 12,476 & 2,720 & 21,8 & 0,41 \\
\hline
\end{tabular}


QUADRO II

ALTURA DA PLANTA - (cm)

\begin{tabular}{|c|c|c|c|c|c|c|c|c|c|c|c|}
\hline $\mathrm{N}$ & $\mathrm{n}$ & $\overline{\mathrm{v}}$ & $\sigma$ & $\sigma \%$ & $\sigma: V_{--}^{-}$ & $\mathrm{N}$ & $\mathrm{n}$ & $\bar{v}$ & $\sigma$ & $\sigma \%$ & $\sigma: / \overline{\bar{v}}$ \\
\hline 559 & 14 & 52,14 & 11,23 & 21,54 & 1,56 & \multirow{4}{*}{$\begin{array}{l}537 \\
539 \\
511 \\
556 \\
528 \\
504\end{array}$} & \multirow{4}{*}{$\begin{array}{l}23 \\
31 \\
27 \\
34 \\
27 \\
31\end{array}$} & 93,04 & \multirow{4}{*}{$\begin{array}{l}19,64 \\
19,39 \\
24,96 \\
11,78 \\
18,45 \\
23,92\end{array}$} & \multirow{4}{*}{$\begin{array}{l}21,11 \\
20,80 \\
26,74 \\
12,59 \\
19,61 \\
25,31\end{array}$} & \multirow{4}{*}{$\begin{array}{l}2,04 \\
2,01 \\
2,00 \\
1,22 \\
1,90 \\
2,46\end{array}$} \\
\hline 554 & 28 & 54,64 & 12,32 & 22,55 & 1,67 & & & 93,23 & & & \\
\hline 577 & 31 & 59,68 & 12,51 & 20,96 & 1,62 & & & 93,53 & & & \\
\hline \multirow{2}{*}{$\begin{array}{l}507 \\
505 \\
555\end{array}$} & \multirow{2}{*}{$\begin{array}{l}33 \\
29 \\
32\end{array}$} & \multirow{2}{*}{$\begin{array}{l}68,18 \\
68,97 \\
69,06\end{array}$} & \multirow{2}{*}{$\begin{array}{l}16,48 \\
11,75 \\
11,46\end{array}$} & \multirow{2}{*}{$\begin{array}{l}24,17 \\
17,04 \\
16,59\end{array}$} & \multirow{2}{*}{$\begin{array}{l}2,00 \\
1,41 \\
1,38\end{array}$} & & & $\begin{array}{l}94,07 \\
94,52\end{array}$ & & & \\
\hline & & & & & & \multirow{6}{*}{$\begin{array}{l}503 \\
527 \\
567 \\
570 \\
562 \\
506 \\
538\end{array}$} & \multirow{6}{*}{$\begin{array}{l}31 \\
31 \\
29 \\
29 \\
34 \\
28 \\
30\end{array}$} & 95,16 & \multirow{6}{*}{$\begin{array}{l}18,23 \\
20,39 \\
18,06 \\
15,67 \\
20,43 \\
18,53 \\
21,16\end{array}$} & \multirow{6}{*}{$\begin{array}{l}19,16 \\
21,07 \\
18,51 \\
16,00 \\
20,86 \\
18,73 \\
21,30\end{array}$} & \multirow{6}{*}{$\begin{array}{l}1,87 \\
2,07 \\
1,83 \\
1,58 \\
2,06 \\
1,86 \\
2,12\end{array}$} \\
\hline 518 & 31 & 70,32 & 13,54 & 19,25 & 161 & & & $\begin{array}{l}96,17 \\
97,59\end{array}$ & & & \\
\hline 561 & 35 & 70,57 & 13,92 & 19,73 & 1,66 & & & 97,93 & & & \\
\hline 560 & 29 & 70,69 & 11,32 & 16,01 & 1,35 & & & 97,94 & & & \\
\hline 551 & 25 & 73,60 & 15,78 & 21,44 & 1,84 & & & 98,93 & & & \\
\hline 568 & 30 & 74,67 & 20,13 & & 2,33 & & & 99,33 & & & \\
\hline 564 & 18 &, 56 & 21,49 & 28,44 & 2,47 & 533 & 21 & 100,48 & 23,97 & 23,86 & 2,39 \\
\hline 522 & 32 & 5,94 & 16,63 & 21,90 & 1,91 & 509 & 31 & & 21 , & 21 & 2,14 \\
\hline 510 & 32 & 81 & 18,45 & 23,71 & 2,09 & 572 & 31 & 102,26 & 17,07 & 16,69 & 1,69 \\
\hline 552 & 33 & 18 & 22,56 & 28,86 & 2,55 & 493 & 35 & 103,14 & 18,11 & 17,56 & 1,78 \\
\hline \multirow[t]{2}{*}{521} & 30 & 00 & 19,3 & 24,48 & 2,18 & \multirow{2}{*}{$\begin{array}{l}550 \\
529\end{array}$} & 34 & 103,53 & 9,50 & 9,50 & 0,93 \\
\hline & \multirow{17}{*}{$\begin{array}{l}23 \\
31 \\
33 \\
32 \\
28 \\
25 \\
15 \\
25 \\
20 \\
22 \\
32 \\
29 \\
32 \\
29 \\
21 \\
28\end{array}$} & & & & & & 12 & 105,00 & 23,16 & 22,06 & 2,26 \\
\hline \multirow{16}{*}{$\begin{array}{l}575 \\
579 \\
557 \\
571 \\
565 \\
574 \\
515 \\
563 \\
534 \\
578 \\
508 \\
576 \\
520 \\
566 \\
535 \\
517\end{array}$} & & 80,43 & 11,40 & 10 & 1,95 & & & & & & \\
\hline & & 80,65 & 17,50 & 21,70 & 1,95 & 530 & 5 & 106,00 & 11,40 & & 1,11 \\
\hline & & 80,91 & 15,08 & 18,64 & 1,68 & 526 & 24 & 106,25 & 23,74 & 34 & 2,30 \\
\hline & & 80,94 & 17,11 & 21,14 & 1,90 & 491 & 34 & 106,47 & 17,84 & 18,63 & 1,73 \\
\hline & & 81,43 & 13,25 & 16,27 & 1,47 & 532 & 30 & 107,33 & 17,21 & 16,03 & 1,66 \\
\hline & & 82,00 & 14,14 & 17,24 & 1,56 & 524 & 24 & 107,50 & 18,24 & 16,97 & 1,76 \\
\hline & & 82,00 & 14,74 & 17,98 & 1,63 & 536 & 30 & 107,67 & 23,15 & 21,50 & 2,23 \\
\hline & & 82,40 & 16,90 & 20,51 & 1,86 & 573 & 28 & 108,21 & 22,29 & 20,60 & 2,14 \\
\hline & & 83,00 & 22,50 & & 2,47 & 483 & 35 & 0,29 & 20,07 & 18,20 & 1,91 \\
\hline & & 83,18 & 20,09 & & 2,20 & 482 & 36 &, 56 & 20,83 & 18,84 & 1,98 \\
\hline & & 83,44 & 14,05 & 16 & 1,54 & 492 & 36 & 17 & 19,48 & 17 , & 1,82 \\
\hline & & 83,45 & 22,88 & & 2,50 & 490 & 36 & 117 & 19,48 & 17,06 & 1,82 \\
\hline & & 84,06 & & & & & & & & & \\
\hline & & 8429 & & & $\begin{array}{l}1,00 \\
230 \\
130\end{array}$ & & & & & & \\
\hline & & 84,64 & 19,53 & & & 497 & 16 & $1]$ & & & 1 , \\
\hline & & & & & & 494 & 36 & & 19, & & \\
\hline 519 & 35 & 85,14 & 19,00 & & 2,06 & & & & & & \\
\hline 512 & 16 & & & & 2,08 & 485 & 35 & 120,86 & 18,53 & 15,33 & 1,69 \\
\hline 502 & 30 & 87,10 & 2572 & & $\begin{array}{l}1,14 \\
2.76\end{array}$ & 484 & 35 & 12 & & & 2,0 \\
\hline & 32 & & 15,50 & & 1,66 & 498 & 36 & & & & 2,0 \\
\hline & & & & & & 487 & 37 & 128,38 & 24,25 & 18,86 & 2,14 \\
\hline & & 90,00 & 35,21 & 39,12 & 3,71 & 495 & 34 & & & & 1,69 \\
\hline 516 & 33 & & & & 10 & 489 & 36 & 132,22 & 21,53 & & 1,87 \\
\hline & 32 & & & & 1,2 & 500 & 17 & & & & 1,22 \\
\hline & 36 & & & & 1,98 & 488 & 35 & 13 & 19 & 14 & 1,68 \\
\hline & 35 & & & & 1,67 & 481 & 36 & & 25,10 & 18,40 & 2,15 \\
\hline & & $\subseteq 2,22$ & 19,22 & 84 & $2,00^{\circ}$ & 486 & 36 & 9,17 & 19,03 & & 1,61 \\
\hline & & & & & & 490 & & & 19,24 & 13,80 & \\
\hline & & & & & & 480 & 35 & 145,14 & 20,64 & 14,22 & 1,71 \\
\hline
\end{tabular}




\section{QUADRO III}

MILHO

Florescimento (dias) da Planta

\begin{tabular}{|c|c|c|c|c|c|c|c|c|c|c|c|}
\hline $\mathrm{N}$ & $n$ & $\overline{\mathrm{v}}$ & $\sigma$ & $\approx \%$ & $\sigma: \sqrt{\tilde{\hat{v}}}$ & $\mathrm{~N}$ & $n$ & $\dddot{\mathrm{v}}$ & $\sigma$ & $\sigma \%$ & $\sigma: \sqrt{\bar{v}}$ \\
\hline 488 & 35 & 46,69 & 2,31 & 4,94 & 0,34 & 499 & 18 & 59,33 & 5,75 & 9,69 & 0,75 \\
\hline 483 & 35 & 47,03 & 3,63 & 7,72 & 0,53 & 518 & 31 & 59,35 & 6,96 & 11,73 & 0,90 \\
\hline \multirow[t]{2}{*}{555} & 32 & 47,50 & 2,64 & 5,56 & 0,38 & 539 & 31 & 59,45 & 4,03 & 6,78 & 0,52 \\
\hline & \multirow{9}{*}{$\begin{array}{l}35 \\
34 \\
36 \\
36 \\
35 \\
36 \\
28 \\
35 \\
34\end{array}$} & \multirow{9}{*}{$\begin{array}{l}47,63 \\
47,85 \\
47,92 \\
48,25 \\
48,49 \\
49,33 \\
49,43 \\
49,43 \\
49,94\end{array}$} & \multirow{9}{*}{$\begin{array}{l}3,03 \\
2,66 \\
2,82 \\
3,47 \\
2,36 \\
3,42 \\
9,15 \\
3,26 \\
4,47\end{array}$} & \multirow{10}{*}{$\begin{array}{r}6,36 \\
5,56 \\
5,88 \\
7,19 \\
4,87 \\
6,93 \\
18,51 \\
6,60 \\
8,95\end{array}$} & \multirow{5}{*}{\multicolumn{2}{|c|}{\begin{tabular}{l||l}
0,45 & 509 \\
0,38 \\
0,41 \\
0,50 \\
0 \\
0
\end{tabular} \mid $\begin{array}{l}533 \\
538 \\
538\end{array}$}} & \multirow{5}{*}{$\begin{array}{l}30 \\
31 \\
21 \\
33 \\
30\end{array}$} & & \multirow{4}{*}{$\begin{array}{l}5,82 \\
5,47 \\
5,12 \\
4,11 \\
6,29\end{array}$} & $\begin{array}{l}9,78 \\
9,19\end{array}$ & \multirow{5}{*}{$\begin{array}{l}0,75 \\
0,71 \\
0,66 \\
0,53 \\
0,81\end{array}$} \\
\hline $\begin{array}{l}492 \\
491\end{array}$ & & & & & & & & $\begin{array}{l}59,54 \\
5957\end{array}$ & & $\begin{array}{r}9,19 \\
027\end{array}$ & \\
\hline 482 & & & & & & & & 59,64 & & $\begin{array}{r}6,21 \\
6,89\end{array}$ & \\
\hline 490 & & & & & & & & 59,70 & & 10,54 & \\
\hline 493 & & & & & 0,34 & & & & & & \\
\hline 489 & & & & & \multirow{4}{*}{$\begin{array}{l}0,49 \\
1,30 \\
0,46 \\
0,63\end{array}$} & \multirow{12}{*}{$\begin{array}{l}524 \\
519 \\
563 \\
567 \\
500 \\
529 \\
527 \\
520 \\
562 \\
517 \\
579\end{array}$} & \multirow{12}{*}{$\begin{array}{l}24 \\
35 \\
25 \\
29 \\
17 \\
12 \\
31 \\
32 \\
34 \\
28 \\
31\end{array}$} & 60,12 & \multirow{11}{*}{$\begin{array}{l}3,48 \\
6,42 \\
3,92 \\
4,46 \\
5,07 \\
7,97 \\
4,48 \\
5,38 \\
5,68 \\
6,70 \\
4,81\end{array}$} & 5,79 & \multirow{12}{*}{$\begin{array}{l}0,45 \\
0,83 \\
0,50 \\
0,57 \\
0,65 \\
1,02 \\
0,57 \\
0,68 \\
0,72 \\
0,85 \\
0,61\end{array}$} \\
\hline 554 & & & & & & & & 60,40 & & 10,63 & \\
\hline 480 & & & & & & & & 60,88 & & 6,44 & \\
\hline 550 & & & & & & & & 61,21 & & 7,29 & \\
\hline & & & & & & & & 61,35 & & 8,26 & \\
\hline 495 & 34 & 50,06 & 2,20 & 4,39 & 0,31 & & & 61,50 & & 12,96 & \\
\hline 485 & 35 & 50,29 & 4,13 & 8,21 & 0,58 & & & 61,58 & & 7,28 & \\
\hline 481 & 36 & 50,42 & 4,45 & 8,83 & 0,63 & & & 62,22 & & 8,65 & \\
\hline 486 & 36 & 50,58 & 3,08 & 6,09 & 0,43 & & & 62,24 & & 9,13 & \\
\hline 487 & 37 & 50,70 & 3,78 & 7,46 & 0,53 & & & 62,28 & & 10,76 & \\
\hline \multirow{8}{*}{$\begin{array}{l}484 \\
502 \\
556 \\
508 \\
496 \\
559 \\
505\end{array}$} & 33 & 50,72 & 4,11 & 8,10 & 0,58 & & & 62,29 & & 7,72 & \\
\hline & \multirow{7}{*}{$\begin{array}{l}35 \\
31 \\
34 \\
32 \\
35 \\
14 \\
29\end{array}$} & \multirow{7}{*}{$\begin{array}{l}50,80 \\
50,84 \\
50,94 \\
51,06 \\
51,31 \\
51,57 \\
52,42\end{array}$} & \multirow{7}{*}{$\begin{array}{l}4,38 \\
4,63 \\
4,49 \\
9,53 \\
3,26 \\
8,72 \\
2,86\end{array}$} & \multirow{7}{*}{$\begin{array}{r}8,62 \\
9,11 \\
8,81 \\
6,91 \\
6,35 \\
16,91 \\
5,45\end{array}$} & \multirow{7}{*}{$\begin{array}{l}0,61 \\
0,65 \\
0,63 \\
1,33 \\
0,46 \\
1,21 \\
0,40\end{array}$} & & & & & & \\
\hline & & & & & & 522 & 32 & 62,59 & 4,17 & 6,66 & 0,53 \\
\hline & & & & & & 566 & 29 & 63,03 & 5,07 & 8,04 & 0,64 \\
\hline & & & & & & 523 & 36 & 63,25 & 5,14 & 8,13 & 0,65 \\
\hline & & & & & & 578 & 22 & 63,73 & 6,06 & 9,51 & 0,76 \\
\hline & & & & & & 528 & 27 & 64,00 & 5,20 & 5,20 & 0,65 \\
\hline & & & & & & 521 & 30 & 64,30 & 6,32 & 9,83 & 0,79 \\
\hline 510 & 32 & & 5.4 & & 0.75 & 565 & 28 & & & & $\begin{array}{l}0,97 \\
061\end{array}$ \\
\hline 504 & 31 & 94 & 7,73 & 14,60 & 1,06 & 580 & & 64,67 & 6,6 & 10,21 & 0,82 \\
\hline 557 & 33 & 53,82 & 3,43 & 6,37 & 0,47 & 574 & 25 & 64,72 & & 7,94 & 0,64 \\
\hline 513 & 32 & 96 & 3,78 & 7,00 & 0,51 & 571 & 32 & 64,75 & 5, & 9,19 & 0,74 \\
\hline 514 & 31 & 03 & 7,84 & 14,51 & 1,07 & 569 & 31 & 64,87 & 5,65 & 8,71 & 0,70 \\
\hline 560 & 29 & 17 & 4,87 & 8,82 & 0,66 & & & & & & \\
\hline 494 & 36 & & 2,89 & 5,30 & 0,40 & 526 & 24 & & 6,73 & 10,33 & 0,83 \\
\hline 553 & 35 & & 4,31 & 7,90 & 0,58 & 568 & 30 & 65,30 & 5,61 & 8,59 & 0,69 \\
\hline 506 & 28 & & 3,68 & 6,25 & 0,5 & 531 & 6 & 65,50 & 3,33 & 5,08 & 0,41 \\
\hline 558 & 32 & & 6,7 & 12,22 & 0,9 & 570 & 29 & 65,55 & 5.07 & 7,73 & 0,63 \\
\hline 498 & 36 & 55 & 4,8 & 8,6 & 0,6 & & 29 & & & 11,23 & 0,91 \\
\hline 515 & 15 & 56,40 & 7,60 & 13,48 & 1,0 & 50 & 26 & & & 9,62 & 0,78 \\
\hline 503 & 31 & 56,64 & 7,82 & 13,81 & 1,0 & 564 & 18 & 67, & & 12,00 & 0,98 \\
\hline 551 & 25 & 56,68 & 5,41 & 9,54 & 0,7 & 575 & 23 & 67,13 & & 7,91 & 0,65 \\
\hline $\begin{array}{l}4 \\
5\end{array}$ & $\begin{array}{l}10 \\
27\end{array}$ & & $\begin{array}{l}4,24 \\
4,78\end{array}$ & $\begin{array}{l}1,48 \\
8,39\end{array}$ & $\begin{array}{l}0,50 \\
0,63\end{array}$ & 573 & 28 & & & & \\
\hline & & & & & & & 20 & 68,35 & & 9,71 & 0,80 \\
\hline $\begin{array}{l}537 \\
516\end{array}$ & 23 & 57,61 & 5,74 & 9,96 & 0,7 & & & & & & \\
\hline 532 & $\begin{array}{l}33 \\
30\end{array}$ & & 5,1 & $\begin{array}{l}8,80 \\
8.87\end{array}$ & 0,6 & $\begin{array}{l}525 \\
535\end{array}$ & 25 & & & & 0,7 \\
\hline & 35 & 58 & 6,05 & 10,35 & 0,79 & 572 & 31 & 72,03 & 4,13 & $\begin{array}{l}0,01 \\
5,73\end{array}$ & 0.49 \\
\hline & 16 & 59,31 & 7,82 & 13,18 & 1.02 & & & & & & \\
\hline
\end{tabular}




\section{QUADRO IV}

VARIAÇÃO dos f́ndices da Variança $\sigma: \sqrt{\mathrm{v}}$

\begin{tabular}{|c|c|c|c|c|c|c|c|c|}
\hline \multirow{2}{*}{ EXPERIÊNCIA } & \multicolumn{3}{|c|}{ TOTAL } & \multicolumn{3}{|c|}{ ENTRE } & \multicolumn{2}{|c|}{ RESIDUO } \\
\hline & $\mathrm{nf}$ & $\sigma$ & $\vartheta$ & $\mathrm{nf}$ & $\sigma$ & $\vartheta$ & nf & $\sigma$ \\
\hline Café & & & & & & & & \\
\hline $\begin{array}{l}\text { Produção em kg } \\
\sigma: \sqrt{\overline{\mathrm{v}}}=1,20\end{array}$ & 35 & 0,31 & 1,15 & 10 & 0,40 & 1,47 & 25 & 0,27 \\
\hline Laranja I & & & & & & & & \\
\hline Número de Frutas & 83 & 1,32 & 1,05 & 14 & 1,64 & 1,31 & 69 & 1,25 \\
\hline Laranja II & & & & & & & & \\
\hline Número de Frutas & 35 & 1,89 & 1,09 & 8 & 2,32 & 1,33 & 27 & 1,74 \\
\hline Milho & & & & & & & & \\
\hline Altura da espiga $(\mathrm{cm})$ & 90 & 0,53 & 0,98 & 7 & 0,51 & 0,94 & 83 & 0,54 \\
\hline Milho & & & & & & & & \\
\hline F'lorescimento (dia) & 90 & 0,23 & 0,99 & 10 & 0,23 & 0,99 & 80 & 0,23 \\
\hline Milho & & & & & & & & \\
\hline Altura da planta $(\mathrm{cm})$ & 90 & 0,42 & 1,17 & 16 & 0,61 & 1,69 & 83 & 0,36 \\
\hline$\sigma: \sqrt{\bar{v}}=1,92$ & & & & & & & & \\
\hline
\end{tabular}




\section{GRÁFICO 1}
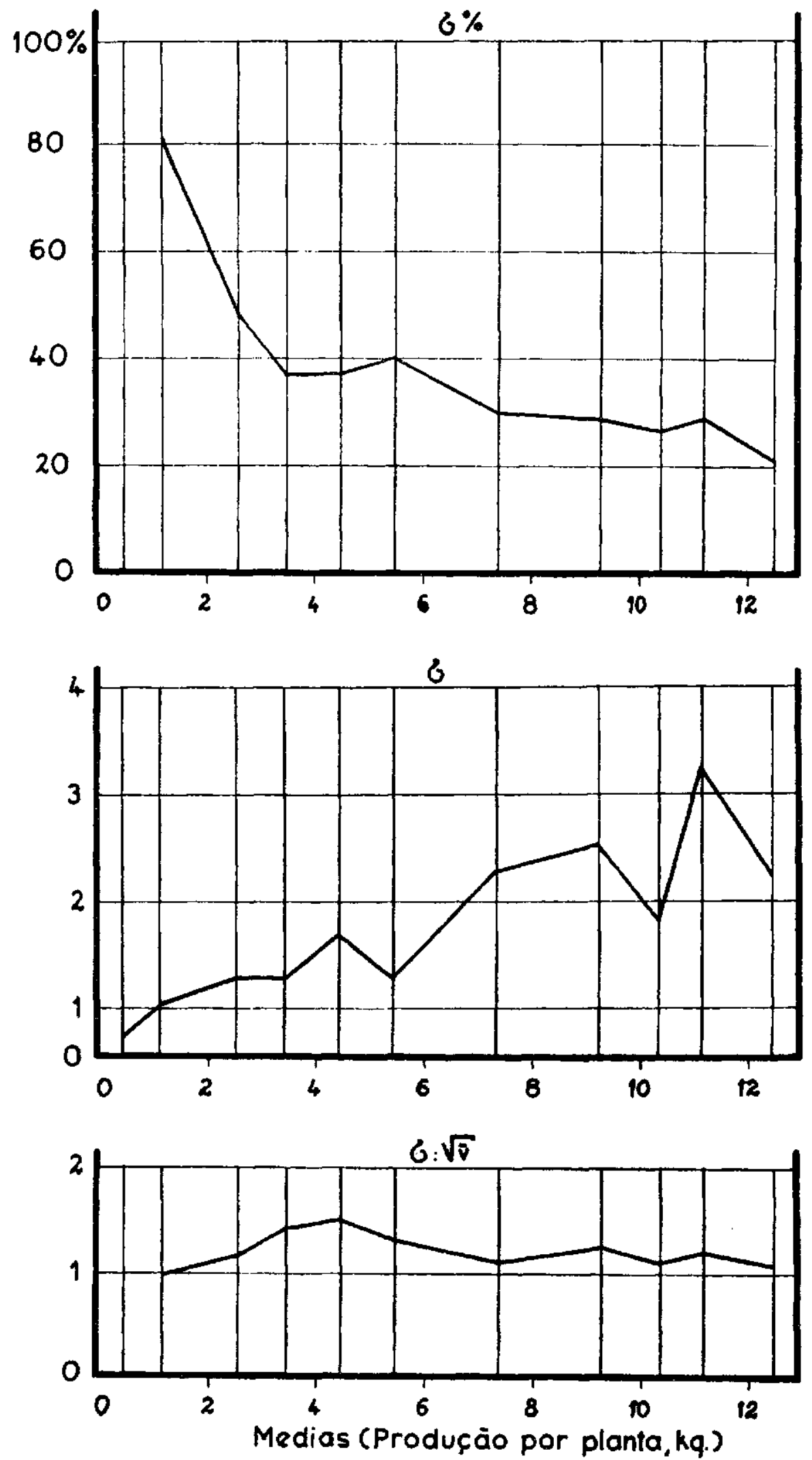

CAFÉ 


\section{GRÁFICO 2}
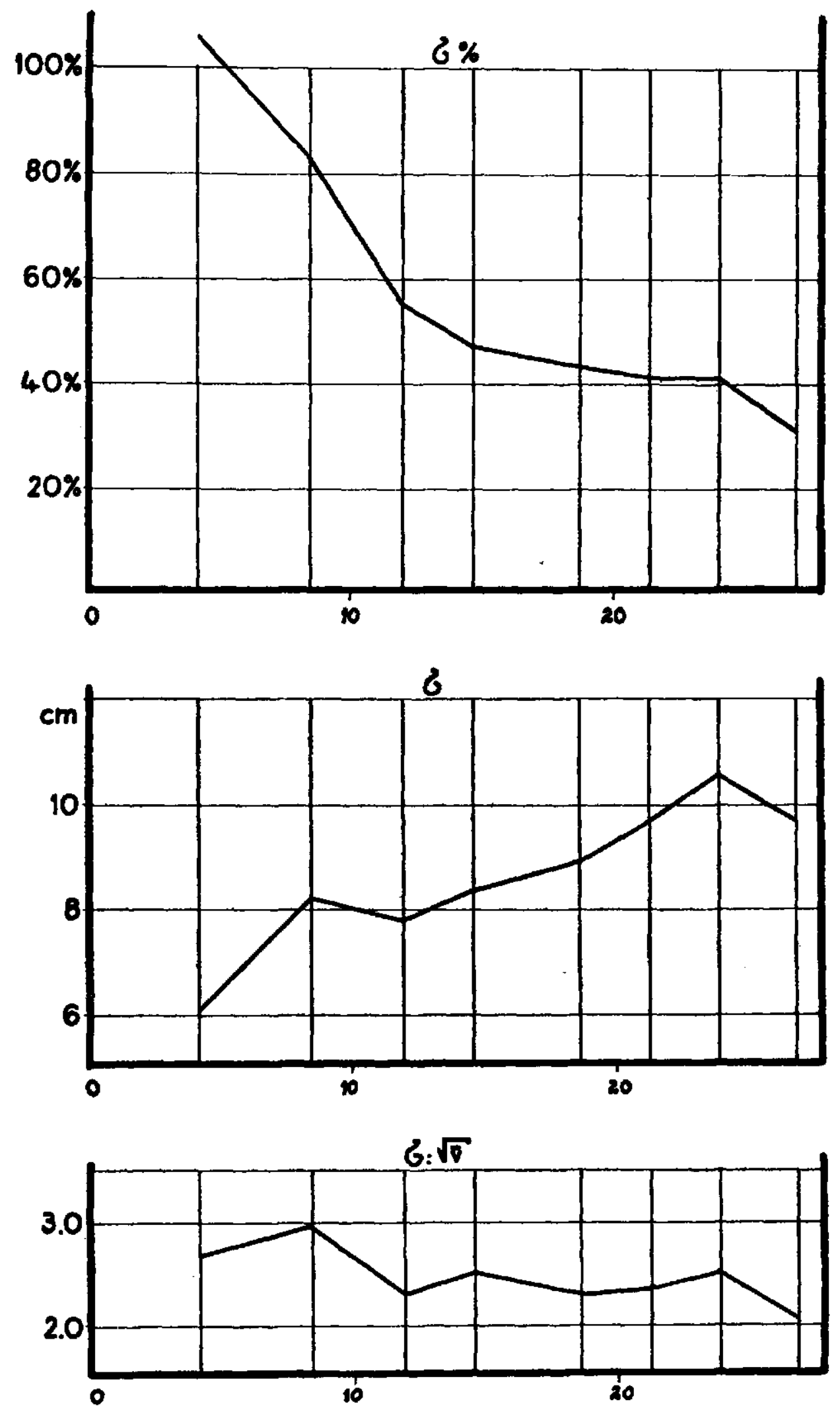

Medias (Altura da espiga, cm) 


\section{GRÁFICO 3}
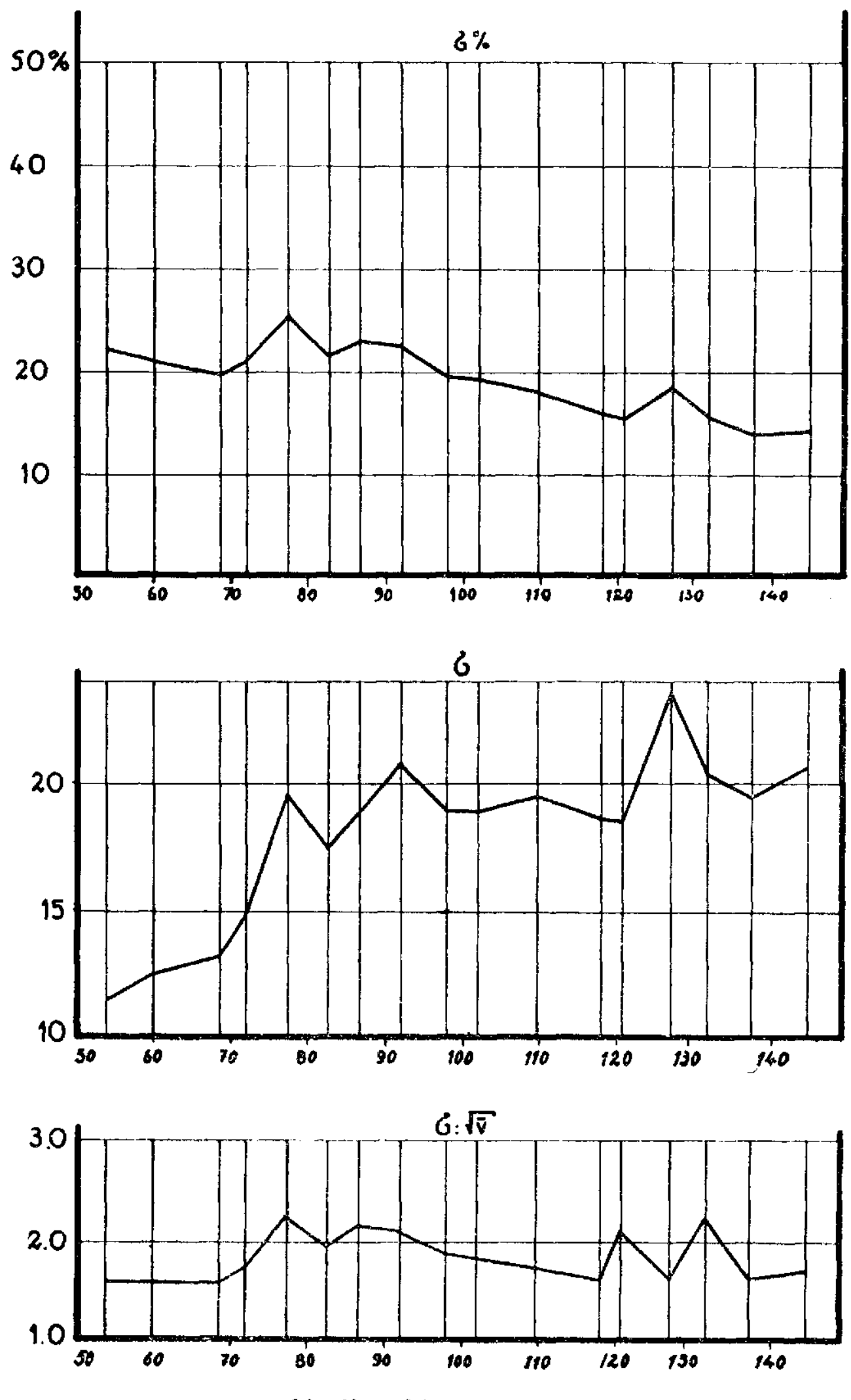

Medias (Allura da planta, cm) 

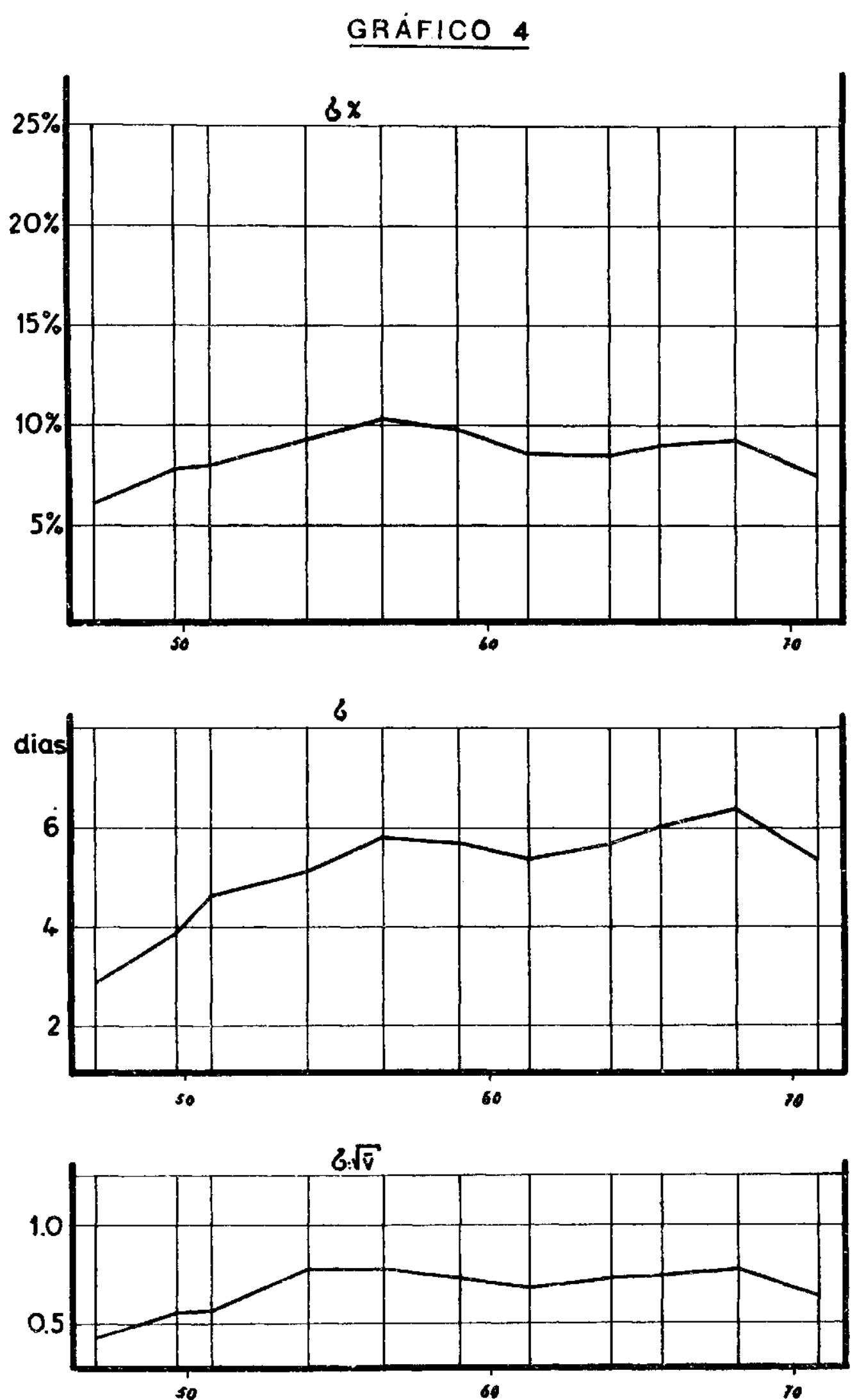

Medias (Florescimento, dias) 

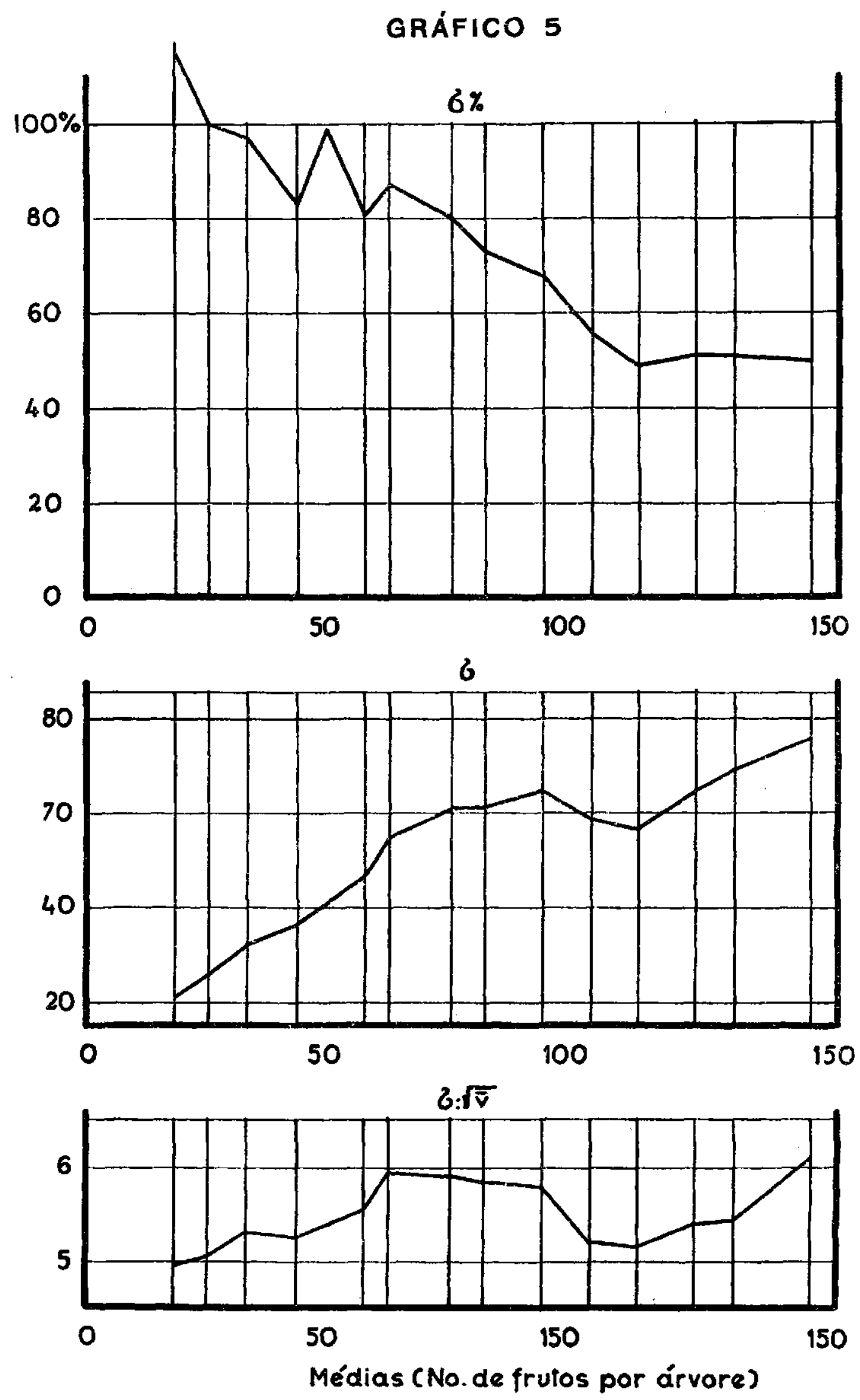

LARANJA (Baianinha) 

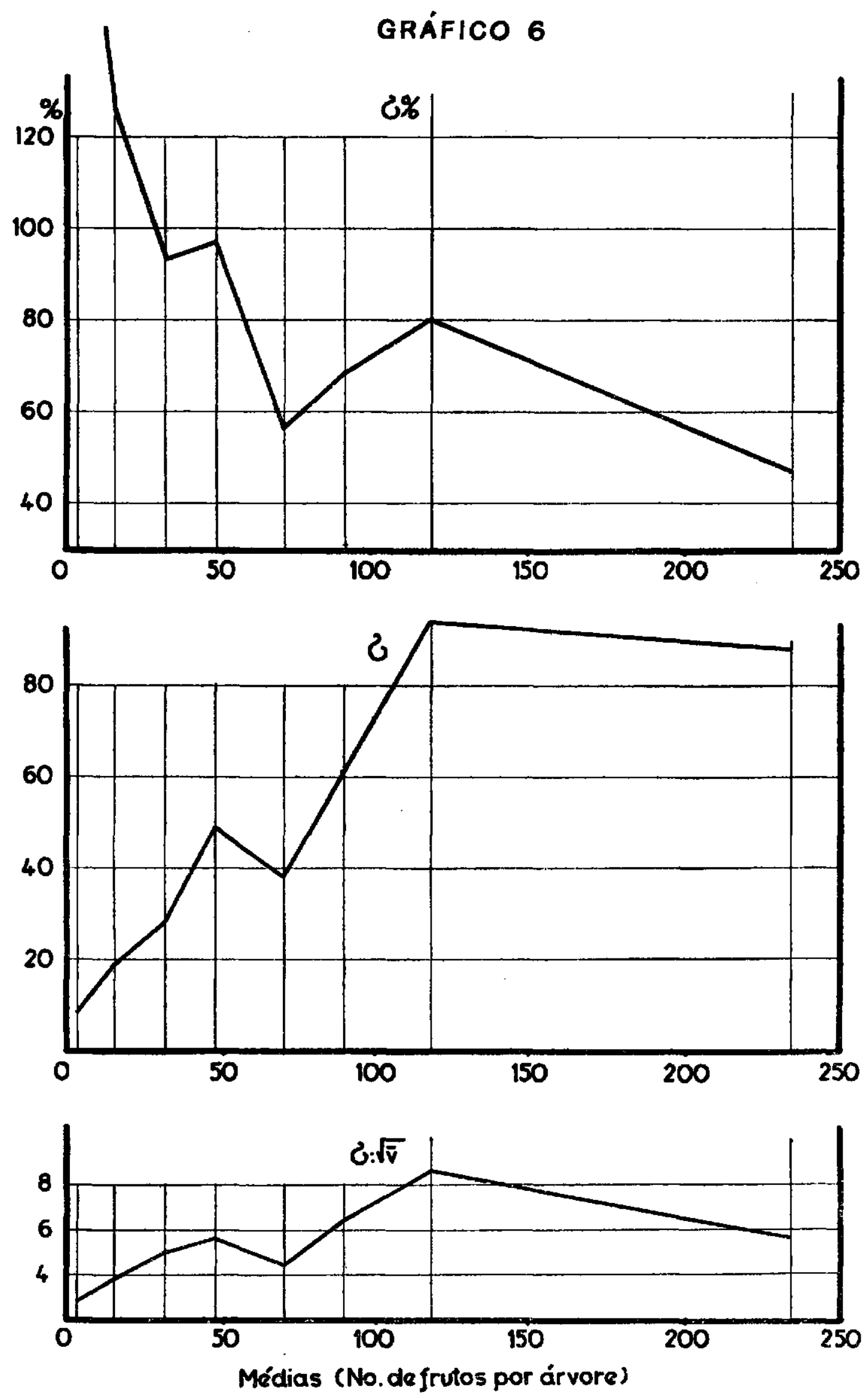

LARANJA (Cavalo-Cavaleiro) 